\title{
Micromagnetic Simulation of Vortex Development in Magnetic Bi-Material Bow-Tie Structures
}

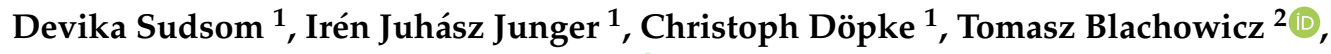 \\ Lothar Hahn ${ }^{3}$ and Andrea Ehrmann 1,*D \\ 1 Faculty of Engineering Sciences and Mathematics, Bielefeld University of Applied Sciences, 33619 Bielefeld, \\ Germany; devika.sudsom@fh-bielefeld.de (D.S.); iren.juhasz_junger@fh-bielefeld.de (I.J.J.); \\ christoph.doepke@fh-bielefeld.de (C.D.) \\ 2 Institute of Physics-Center for Science and Education, Silesian University of Technology, 44-100 Gliwice, \\ Poland; tomasz.blachowicz@polsl.pl \\ 3 Karlsruhe Nano Micro Facility (KNMF), Karlsruhe Institute of Technology (KIT), \\ 76344 Eggenstein-Leopoldshafen, Germany; lothar.hahn@kit.edu \\ * Correspondence: andrea.ehrmann@fh-bielefeld.de
}

Received: 1 December 2019; Accepted: 9 January 2020; Published: 12 January 2020

\begin{abstract}
Magnetic vortex structures are of high technological relevance due to their possible application in magnetic memory. Moreover, investigating magnetization reversal via vortex formation is an important topic in basic research. Typically, such vortices are only investigated in homogeneous magnetic materials of diverse shapes. Here, we report for the first time on micromagnetic simulation of vortex formation in magnetic bow-tie nanostructures, comprising alternating parts from iron and permalloy, investigated for two different thicknesses and under different angles of the external magnetic field. While no vortex was found in pure permalloy square, nanoparticles of the dimensions investigated in this study and in case of iron only a relatively thick sample allowed for vortex formation, different numbers of vortices and antivortices were found in the bow-tie structures prepared from both materials, depending on the angular field orientation and the sample thickness. By stabilizing more than one vortex in a confined nanostructure, it is possible to store more than one bit of information in it. Our micromagnetic simulations reveal that such bi-material structures are highly relevant not only for basic research, but also for data storage applications.
\end{abstract}

Keywords: magnetic nanostructures; micromagnetic simulation; bow-tie structure; vortex; magnetization reversal; iron, permalloy; lithography

\section{Introduction}

Magnetic nanostructures can be produced with typical thicknesses of a few nanometers up to tens of $\mathrm{nm}$ and with lateral dimensions in the range of some $10 \mathrm{~nm}$ to several micrometers, applying lithographic techniques [1,2], pulsed electrodeposition [3] or self-assembly methods [4]. Such nanostructures are generally of high interest since the configurational anisotropy due to the shape of the nanoparticles can be in a similar order of magnitude as magneto-crystalline and magneto-elastic anisotropies, giving rise to new effects caused by the superposition of all these anisotropies [5-8].

Magnetic nano-dots were especially investigated by diverse research groups, since they often enable the formation of a vortex which can be used for data storage $[9,10]$. One advantage of such vortex states is the reduction of stray fields and thus of interactions with neighboring nanoparticles [11,12], although such interactions are not fully suppressed [13]. Flux-closed vortex states can be created in round, elliptical or square nano-rings without a core region, again reducing stray fields [14-18], while nano-dots of different shapes with a core region may enable formation of more magnetic states [19-22]. 
A former simulation of round iron $(\mathrm{Fe})$ nanodots revealed nucleation of one or even two vortices during magnetization reversal, depending on the lateral dimensions of the nanodot and its thickness, but also several dimensions and aspect ratios in which no vortex state could be reached [23]. These vortices were often accompanied by antivortices, which are typically unstable and tend to annihilate at the edges of a nanostructure [24]. In square Fe nanodots, seven different magnetization reversal processes were found for different orientations of the external magnetic field, five of which contained one or more vortex states [25]. Similarly, dynamic simulations of rectangular nanostructures showed one or two resonance frequencies for different aspect ratios [26]. While square magnetic nanostructures of pure ferromagnetic or ferromagnetic/antiferromagnetic materials are sometimes reported in the literature [6,27-30], no investigations of bi-material square nanodots have been performed yet to the best of our knowledge.

Here, we report on square bow-tie structures (Figure 1), comprising alternating triangles of iron and permalloy (Py), which show unexpected vortex formation in comparison with square nanodots of both pure materials. Our micromagnetic simulations underline the new possibilities, which may be opened by such bi-material structures in basic research and technological applications. An overview of the possible production of such structures is given in Materials and Methods.
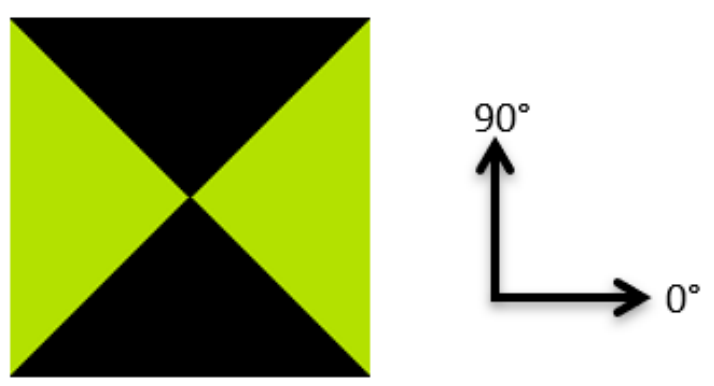

Figure 1. Sketch of the bow-tie structure under examination consisting of iron (black) and permalloy (green) as well as orientation of the external magnetic field angles.

\section{Results and Discussion}

Firstly, Figure 2 depicts typical magnetization states during the reversal process from positive to negative saturation and hysteresis loops of pure Fe nanoparticles of $15 \mathrm{~nm}$ and $30 \mathrm{~nm}$ thickness, respectively. Simulations of the other angles under examinations revealed no qualitative differences and are thus not shown here.

In all graphical representations of the magnetization in the sample, the red color indicates a local magnetization orientation to the right, while the blue color shows magnetic moments oriented to the left. In the hysteresis loops, the longitudinal magnetization component $\mathrm{M}_{\mathrm{L}}$ is defined as parallel to the external magnetic field, while the transverse magnetization component $\mathrm{M}_{\mathrm{T}}$ depicts the hysteresis curve perpendicular to it.

While the Fe particle with thickness $15 \mathrm{~nm}$ shows magnetization reversal via a meander state, as already reported in Ref. 25 and also typical in thin-film systems [31], a vortex state is formed for a thickness of $30 \mathrm{~nm}$, indicated by the "jump" in the hysteresis loop at $-16 \mathrm{mT}$ (Figure 2). The vortex state is here thus only reached after remanence, which can be different for slightly modified field orientations or geometries [25]. 


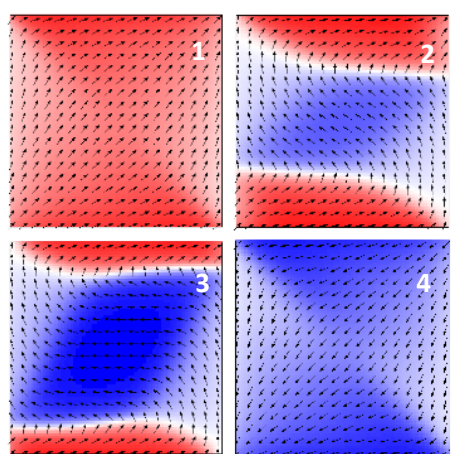

(a)

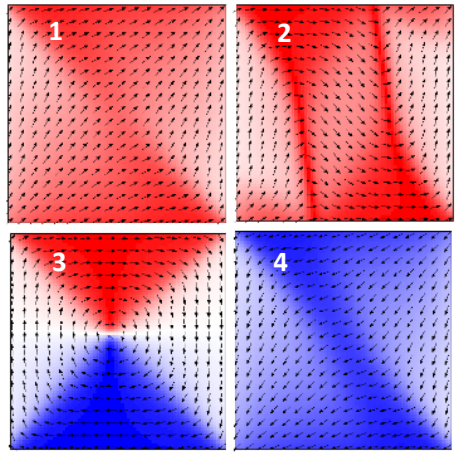

(c)

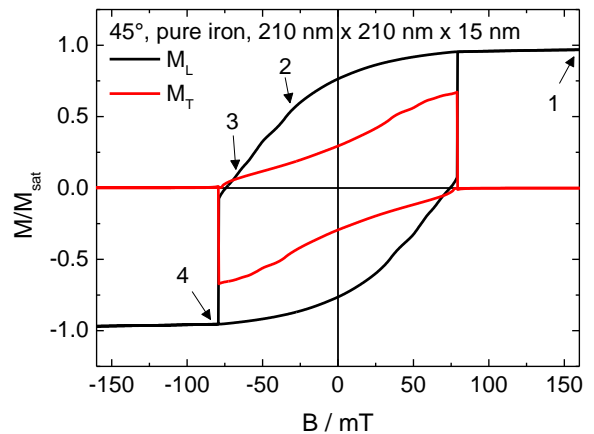

(b)

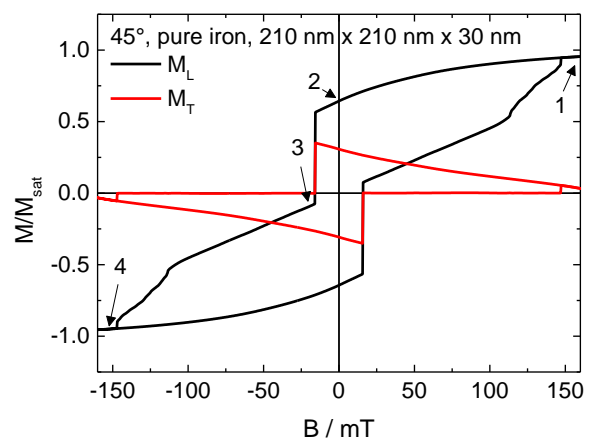

(d)

Figure 2. Snapshots of the magnetization reversal process and hysteresis loops of pure iron nanoparticles, simulated for an external field orientation of $45^{\circ}$, for a thickness of $(\mathbf{a}, \mathbf{b}) 15 \mathrm{~nm}$ or $(\mathbf{c}, \mathbf{d}) 30 \mathrm{~nm}$, respectively. In all graphs, the longitudinal magnetization $\mathrm{M}_{\mathrm{L}}$ is detected parallel to the magnetic field, while the transverse magnetization $\mathrm{M}_{\mathrm{T}}$ is perpendicular to it.

For permalloy, the symmetric $45^{\circ}$ orientation-i.e., the external magnetic field is oriented along $45^{\circ}$, resulting in the same situation as if the sample had been rotated by this angle-shows domain wall states, while the other orientations reveal magnetization reversal via meander states for both thicknesses (Figure 3). The Py nanoparticle does not exhibit any vortex state for the dimensions and field orientations examined in this study.

While three of the four particles modelled from pure materials did not show any vortex states, this changes by forming the bow-tie structure. The following figures depict snapshots of magnetization reversal states and hysteresis loops detected for field orientations of $30^{\circ}, 45^{\circ}$ and $60^{\circ}$, respectively.

Snapshots of the magnetization reversal process for the thinner sample $(15 \mathrm{~nm})$ are depicted in Figure 4a. Here, starting from positive saturation, firstly the typical aforementioned meander state is formed, before a double-vortex state is reached, finally leading to negative saturation. The first "jump" in the hysteresis loops in Figure $4 \mathrm{~b}$ corresponds to the start of this double-vortex state. Generally, such steps along the hysteresis loops indicate the possibility that vortex states or domain walls are nucleated. 


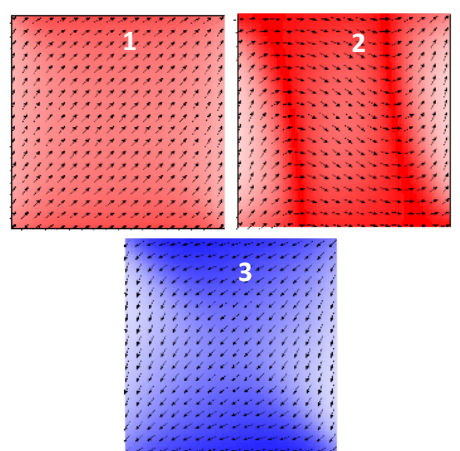

(a)

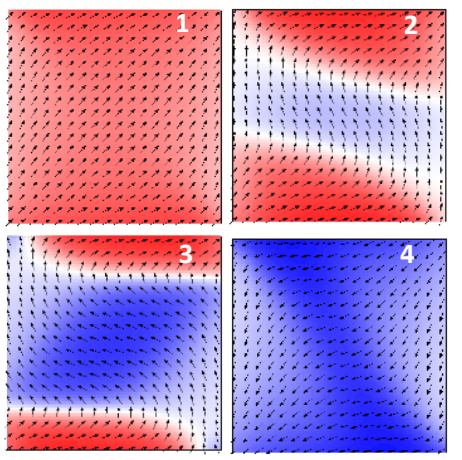

(c)

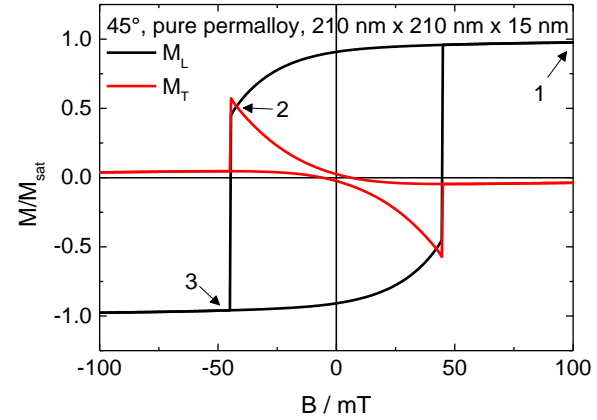

(b)

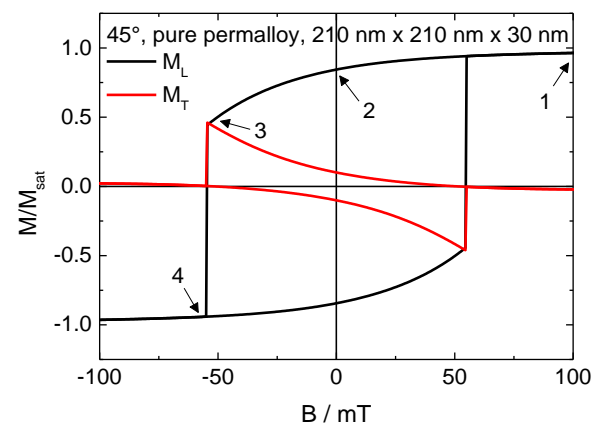

(d)

Figure 3. Snapshots of the magnetization reversal process and hysteresis loops of pure permalloy nanoparticles, simulated for an external field orientation of $30^{\circ}$, for a thickness of $(\mathbf{a}, \mathbf{b}) 15 \mathrm{~nm}$ or (c,d) $30 \mathrm{~nm}$, respectively.

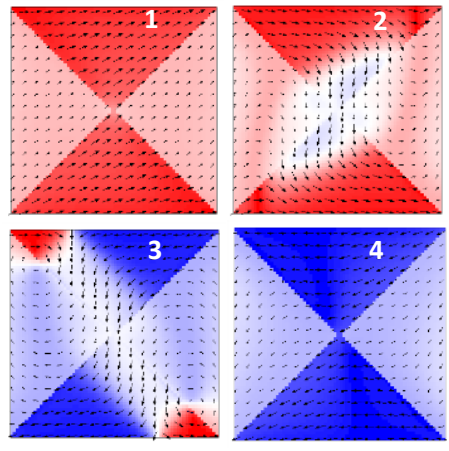

(a)

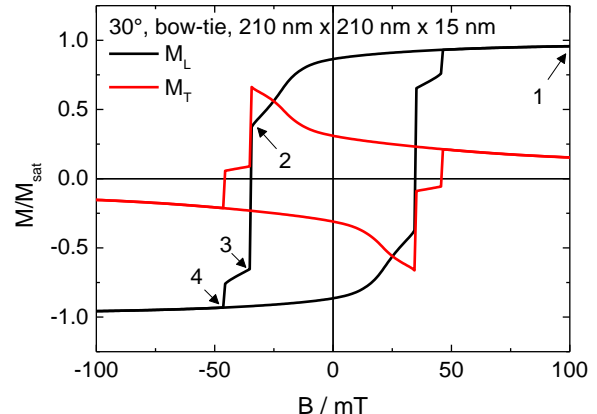

(b)

Figure 4. (a) Snapshots of the magnetization reversal process and (b) hysteresis loops of bow-tie nanoparticles, simulated for an external field orientation of $30^{\circ}$ and a thickness of $15 \mathrm{~nm}$.

For a thickness of $30 \mathrm{~nm}$, magnetization reversal comprises more intermediates states, as visible in the snapshots in Figure $5 \mathrm{a}$ and indicated by the large amount of steps along the slopes of the hysteresis curves for this case (Figure 5b).

Starting again at positive saturation, the already known meander state is formed. Next, a double-vortex state is visible, as it was simulated earlier for pure Fe nanoparticles of similar dimensions [25]. In the next step, two more vortex-antivortex pairs are created which have not yet been found in pure Fe nanodots. While both vortex cores are firstly trapped at the border between Fe and Py, the first vortex pair starts moving into the Fe region now, while the second vortex pair moves slightly into the Py region. It should be mentioned that the newly formed antivortex, opposite to previous literature reports [24], does not visibly move to one of the nanoparticle edges. With the external magnetic field becoming more and more negative, firstly the second vortex-antivortex pairs vanish, before the first vortex pair also vanishes, and magnetization reversal is finished. 

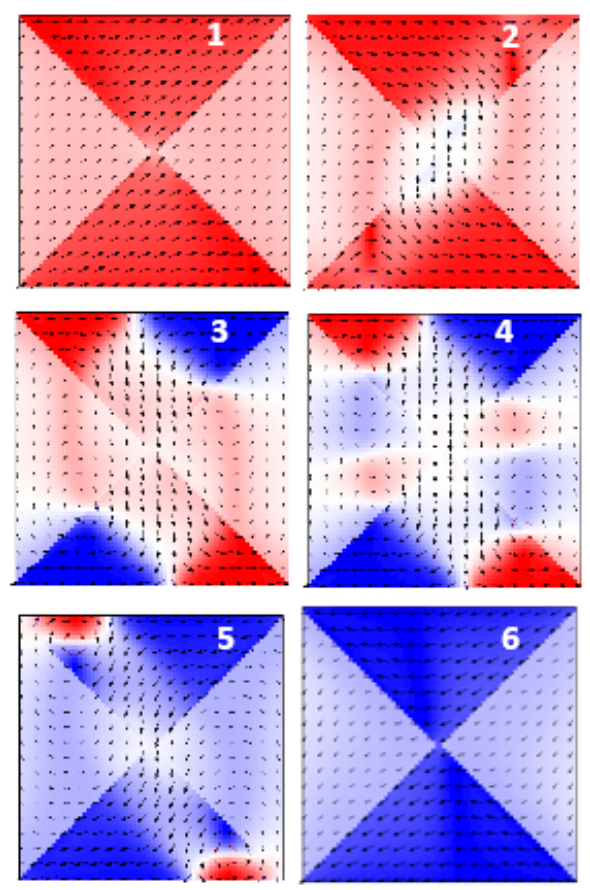

(a)

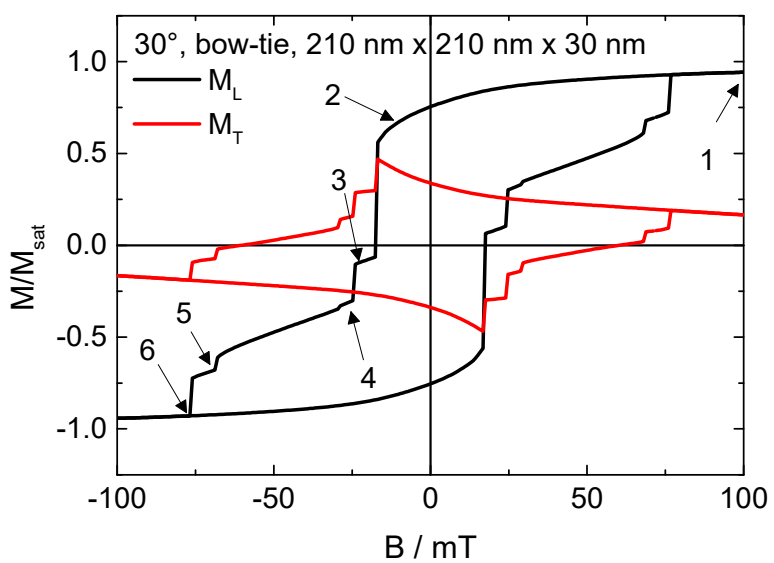

(b)

Figure 5. (a) Snapshots of the magnetization reversal process and (b) hysteresis loops of bow-tie nanoparticles, simulated for an external field orientation of $30^{\circ}$ and a thickness of $30 \mathrm{~nm}$.

For a sample orientation of $45^{\circ}$, there is interestingly no vortex state visible for the thinner sample, which is identical to the pure Fe and Py specimens, respectively (Figure 6).

The thicker sample, however, shows again an interesting magnetization reversal process, as indicated by the corresponding hysteresis loops (Figure 7) and clearly visible in the snapshots of this magnetization reversal process.
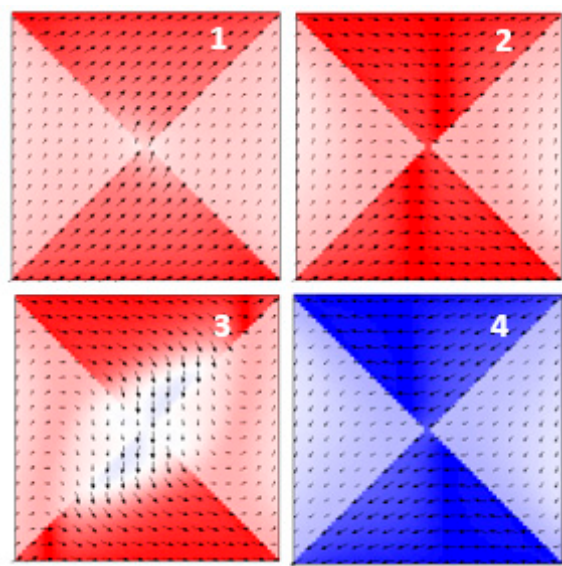

(a)

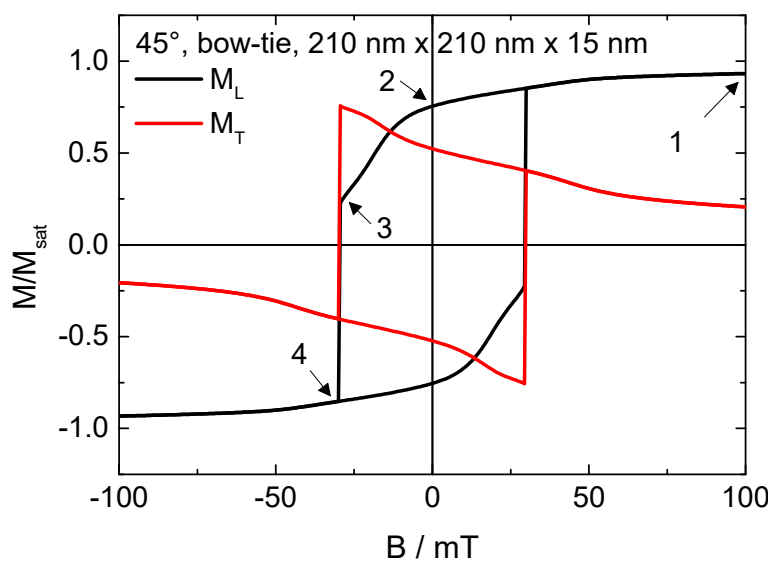

(b)

Figure 6. (a) Snapshots of the magnetization reversal process and (b) hysteresis loops of bow-tie nanoparticles, simulated for an external field orientation of $45^{\circ}$ and a thickness of $15 \mathrm{~nm}$.

Magnetization reversal again starts with a meander state, as seen before, this time leading to nearly fully symmetric vortices in the Fe areas. With the external magnetic field becoming more negative, these vortices start moving to the borders between both materials, while the antivortices propagate along the border from the middle of the Py areas to the middle of the Fe areas where they are more prominently visible due to the here chosen color code. Next, unexpectedly one of the vortices 
vanishes, resulting in an asymmetric situation. Such asymmetries are at first glance unusual, but can be found in many simulations due to small asymmetries of the geometry or the anisotropy distribution. Finally, the single vortex vanishes, and magnetization reversal is completed.
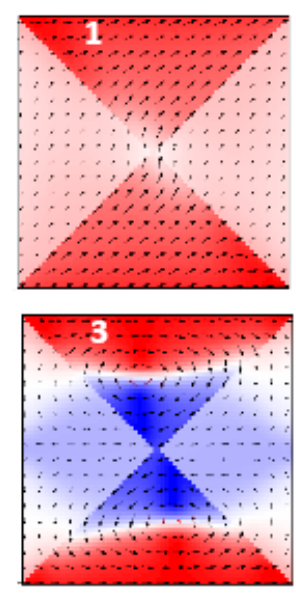

5

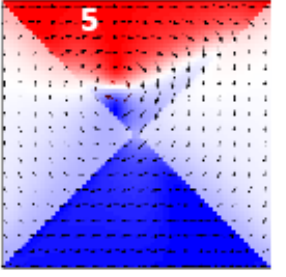

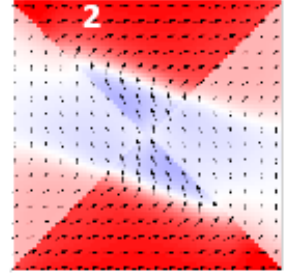

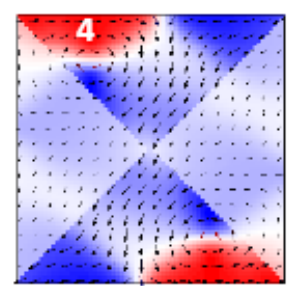

6

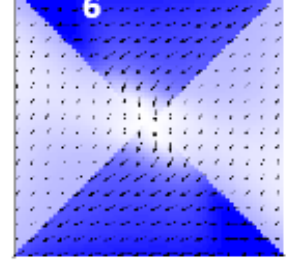

(a)

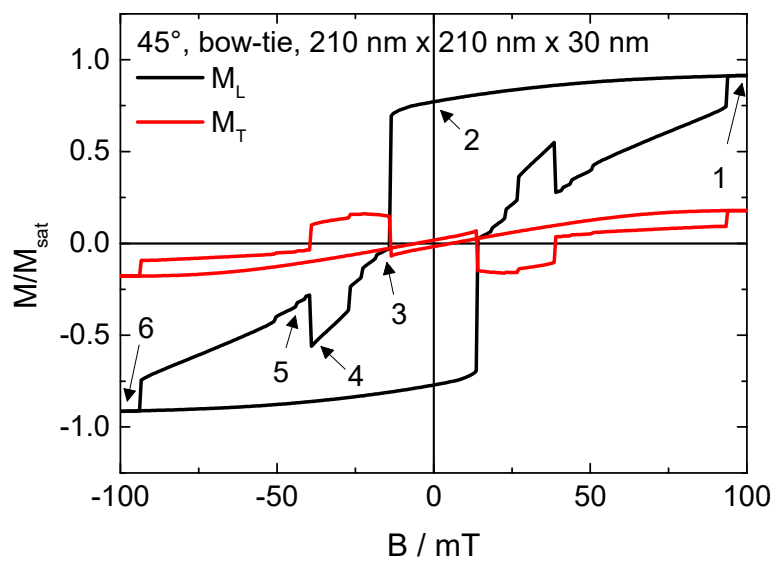

(b)

Figure 7. (a) Snapshots of the magnetization reversal process and (b) hysteresis loops of bow-tie nanoparticles, simulated for an external field orientation of $45^{\circ}$ and a thickness of $30 \mathrm{~nm}$.

Next, Figures 8 and 9 depict snapshots of the magnetization reversal as well as hysteresis loops for an external magnetic field orientation of $60^{\circ}$. For both thicknesses under examination, steps along the loops can be recognized, indicating vortex or domain wall states during magnetization reversal. While the sample of thickness $15 \mathrm{~nm}$ reverses magnetization without vortices, the thicker sample shows a double-vortex very similar to Figure 5 (Figure 9). The nearly identical states during magnetization reversal in the thicker samples for field orientations of $45^{\circ}$ (Figure 7 ) and $60^{\circ}$ (Figure 9) point out the strong influence of the configurational anisotropy, especially for small external magnetic fields counteracting the configurational anisotropy.

Generally, it can be stated that the bow-tie structure fully prevents formation of a vortex core in the middle of the sample. This results in all cases under examination in the formation of a symmetric double-vortex structure, with the vortex cores being formed either in the Fe area or near the border between both materials. 


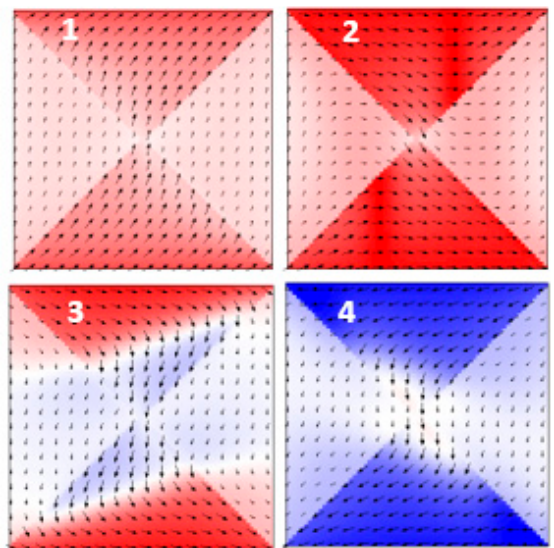

(a)

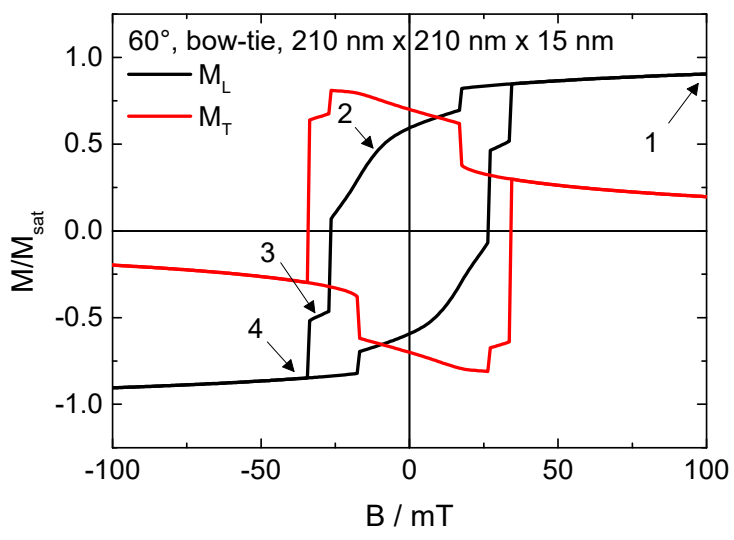

(b)

Figure 8. (a) Snapshots of the magnetization reversal process and (b) hysteresis loops of bow-tie nanoparticles, simulated for an external field orientation of $60^{\circ}$ and a thickness of $15 \mathrm{~nm}$.

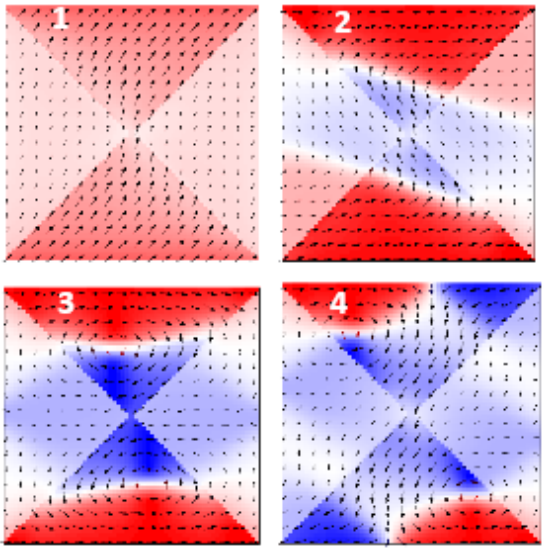

(a)

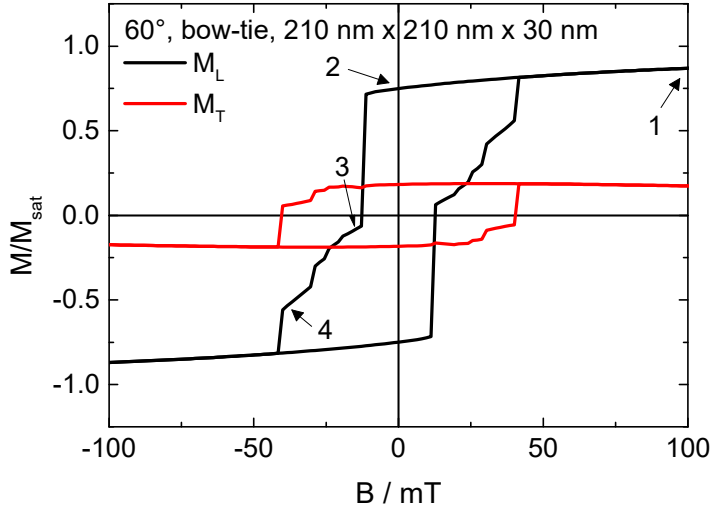

(b)

Figure 9. (a) Snapshots of the magnetization reversal process and (b) hysteresis loops of bow-tie nanoparticles, simulated for an external field orientation of $60^{\circ}$ and a thickness of $30 \mathrm{~nm}$.

Next, to enable storing more than one bit of information in such a structure, it is necessary to break this mirror symmetry by either modifying the thickness or-technologically easier-varying the Fe and Py areas, in this way possibly increasing the number of stable intermediate states with different vortex chirality. It should be mentioned that similar results have also been obtained by Nasirpouri et al., working with pyramidal and truncated icosahedral structures with nickel shell, which the authors also suggested using in multi-bit storage architecture [32].

Another highly interesting topic to be potentially combined with the here suggested bi-material structures are chiral magnets and skyrmions whose interactions with interfaces are of high interest in recent research. This means that instead of using vortices for data storage, as suggested here, skyrmions could be used for this purpose. The materials for such skyrmion-based structures, on the other hand, would be atomically-thin ferromagnets from materials like Fe or Co, typically on heavy-metal substrates like Pt or Pd, instead of the here used much thicker Fe/Py structures. Mulkers et al., e.g., pointed out the possibility to have multiple stable cycloidal states with different periods in laterally confined thin ferromagnetic films with Dzyaloshinskii-Moriya interaction (DMI) as well as stable skyrmions [33]. Even more interesting phenomena are reported by the same group, investigating heterochiral 2D structures, starting with spin canting at interfaces between regions with different DMI to strong domain pinning to improved skyrmion racetrack memory [34]. For the refraction of spin waves at such DMI interfaces in heterochiral magnetic films, a generalized Snell's law was setup which 
depends on an in-plane magnetic field, in this way giving rise to the critical incident angles and showing asymmetric Brewster angles at interfaces between regions with different DMI [35]. Most recently, the group investigated in detail the interaction of skyrmions with heterochiral interfaces and found deflection of the trajectory of ferromagnetic skyrmions which could be tuned by the spin-polarized current applied and the DMI difference on both sides of the interface, while AFM skyrmions were not deflected, but had a much higher speed [36]. Similar investigations should be carried out with the here proposed structures, too.

\section{Materials and Methods}

The nanoparticles under examination in this study are bow-tie structures, as depicted in Figure 1, consisting of Fe and Py parts. Lateral dimensions are $210 \mathrm{~nm} \times 210 \mathrm{~nm}$, the thickness is $15 \mathrm{~nm}$ or $30 \mathrm{~nm}$. Such bow-tie structures are well known as electrodes [37].

There are two methods to produce bow-tie structures by electron beam lithography (EBL). The most common one starts with spin-coating a chip with PMMA resist. The PMMA is structured by EBL. After developing the resist (structures are cavities in the resist), metal is deposited on the substrate by sputtering or evaporation. Redundant metal and resist are removed by a lift-off process. An alternative is to metalize the chip firstly. Then structures are written by EBL and the resist is developed. Now the resist covers the structures and acts as an etch mask. Residual metal is dry etched and the resist is stripped. Bow tie structures remain, as visible in Figure 10.

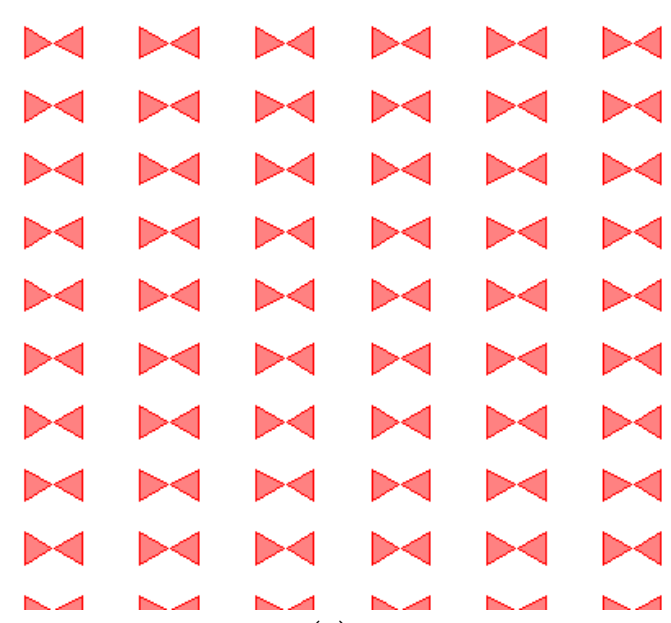

(a)

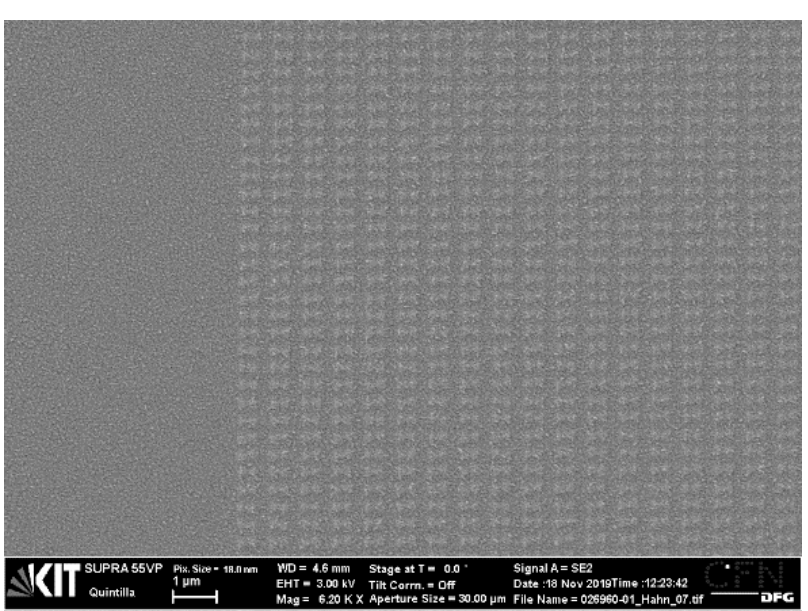

(b)

Figure 10. Single-material bow tie structures (a) as layout and (b) produced with an edge length of 200 $\mathrm{nm}$ with negative resist AR-N.

To obtain bi-material bow tie structures, a so-called bilayer process is necessary, a well-known process for structures with edge lengths of several $100 \mathrm{~nm}$. The alignment precision of the e-beam process is approx. $20 \mathrm{~nm}$, thus with a designed gap of $50 \mathrm{~nm}$ between the materials, a gap in the range of 30-70 nm can be expected. Recently, the writing accuracy as well as the necessary gap between both materials are being optimized.

Our recent simulations concentrate on individual nano-elements, before in a future simulation arrays of these structures will be modeled. Recent simulations of iron nanoparticles revealed that for inter-particle distances similar to the particle diameters, the influence of interactions with neighboring particles is relatively small [38,39], so that simulating individual elements firstly can be expected to be sufficient for a first comparison with the experiment.

The angles of the external magnetic field were chosen as $30^{\circ}, 45^{\circ}$, and $60^{\circ}$; the field is oriented in the plane of the nanostructure. As a comparison, pure Fe and Py nanoparticles of identical dimensions were modelled. 
Simulations were performed with the micromagnetic simulator OOMMF (Object Oriented MicroMagnetic Framework) [40]. In this program, finite differences are used for meshing, and the Landau-Lifshitz-Gilbert (LLG) equation of motion is solved dynamically [41].

OOMMF offers the possibility to create bi-material maps in different ways, using the eXtensible Solver. Here, material regions were defined by an image file (Figure 1), using the function ImageAtlas. The material parameters for $\mathrm{Fe}(\mathrm{Py})$ were chosen according to typical literature values: magnetization at saturation $\mathrm{M}_{\mathrm{S}, \mathrm{Fe}}=1700 \times 10^{3} \mathrm{~A} / \mathrm{m}\left(\mathrm{M}_{\mathrm{S}, \mathrm{Py}}=796 \times 10^{3} \mathrm{~A} / \mathrm{m}\right)$, exchange constant $\mathrm{A}_{\mathrm{Fe}}=21 \times 10^{-12} \mathrm{~J} / \mathrm{m}\left(\mathrm{A}_{\mathrm{Py}}=10.5 \times 10^{-12} \mathrm{~J} / \mathrm{m}\right)$, magneto-crystalline anisotropy constant $\mathrm{K}_{1, \mathrm{Fe}}=48 \times 10^{3} \mathrm{~J} / \mathrm{m}^{3}\left(\mathrm{~K}_{1, \mathrm{Py}}=0.5 \times 10^{3} \mathrm{~J} / \mathrm{m}^{3}\right)$. Along the borders, these values were averaged between the values of the single materials.

It should be mentioned that computing on graphical processing units (GPUs), e.g., with the free GPU-accelerated micromagnetic solver Mumax based on the Nvidia CUDA toolkit, can be used to reach higher performance and can be used for larger-scale, more detailed simulations [42].

The crystallographic orientations in the single parts of the mesh are chosen arbitrarily, as would be the case for a sputtered sample. This means that in each of the cubic "grains", an arbitrary crystallographic orientation is chosen which varies with each new simulation, unlike the larger patches with well-defined differing crystallographic orientations used for simulations of amorphous ferrimagnets [43]. Opposite to the large magneto-crystalline anisotropy of cobalt which causes severe modifications from one simulation to the next for such an approach [1], here subsequent simulations showed approximately identical hysteresis loops which were dominated by the configurational anisotropy and not influenced by the arbitrary crystallographic orientation nor by the starting configuration. The material parameters are constant inside the "grains", no special parameters are taken into account at the grain boundaries inside either material. A quasi-static case was modelled by setting the Gilbert damping constant $\alpha=0.5$ for both materials, the mesh size was chosen as $3 \mathrm{~nm}^{3}$ for the whole sample. Simulations were typically performed in the range between $\pm 200 \mathrm{mT}$ in steps of $0.4 \mathrm{mT}$; if minor loops were found, the field ranges were correspondingly increased to investigate proper full hysteresis loops. All simulations are started from a fully saturated state of the magnetization along the corresponding angle.

\section{Conclusions}

Square magnetic bow-tie nanostructures, comprised of iron and permalloy, were investigated by micromagnetic simulations for the first time. Depending on the orientation with respect to the external magnetic field and on the sample thickness, in most cases two or four vortices were found, while the typical single-vortex state which is often visible in round or square nanodots of different materials and dimensions was fully suppressed by the bi-material structure. These findings underline the opportunities given by such bi-material nanostructures in data storage and transport, allowing for storing more than one bit of information in a nanoparticle which contains more than one separately controllable vortex.

Next, preparation of such bow-tie bi-material structures by e-beam lithography will be optimized, and experimental investigations of the nanostructures will be compared with the simulated results. Finally, simulations and experimental verifications are necessary to evaluate possibilities to influence the vortices solely, in this way enabling storage of more than one bit of information in such a magnetic nanoparticle.

Author Contributions: Conceptualization, D.S. and A.E.; methodology, D.S.; validation, I.J.J., T.B., L.H. and A.E.; formal analysis, D.S., T.B. and A.E.; investigation, D.S.; writing—original draft preparation, A.E., T.B. and L.H.; writing-review and editing, all authors; visualization, D.S., C.D. and A.E. All authors have read and agreed to the published version of the manuscript.

Funding: This work was supported by Volkswagen Foundation grant "Adaptive Computing with Electrospun Nanofiber Networks" no. 93679 and the internal PhD funds of Bielefeld University of Applied Sciences. The APC is funded by the Open Access Publication Fund of Bielefeld University of Applied Sciences and the Deutsche Forschungsgemeinschaft (DFG, German Research Foundation)-414001623. 
Conflicts of Interest: The authors declare no conflict of interest. The funders had no role in the design of the study; in the collection, analyses, or interpretation of data; in the writing of the manuscript, or in the decision to publish the results.

\section{References}

1. Blachowicz, T.; Kosmalska, D.; Döpke, C.; Leiste, H.; Hahn, L.; Ehrmann, A. Varying steps in hysteresis loops of Co square nano-frames. J. Magn. Magn. Mater. 2019, 491, 165619. [CrossRef]

2. Ehrmann, A.; Blachowicz, T.; Komraus, S.; Nees, M.-K.; Jakobs, P.-J.; Leiste, H.; Mathes, M.; Schaarschmidt, M. Magnetic properties of square Py nanowires: Irradiation dose and geometry dependence. J. Appl. Phys. 2015, 117, 173903. [CrossRef]

3. Pagnanelli, F.; Altimari, P.; Bellagamba, M.; Granata, G.; Moscardini, E.; Schiavi, P.G.; Toro, L. Pulsed electrodeposition of cobalt nanoparticles on copper: Influence of the operating parameters on size distribution and morphology. Electrochim. Acta 2015, 155, 228-235. [CrossRef]

4. Romero, M.; Pardo, H.; Faccio, R.; Suescun, L.; Vazquez, S.; Laborda, I.; Fernandez-Werner, L.; Acosta, A.; Castiglioni, J.; Mombru, A.W. A Study on the Polymer Precursor Formation and Microstructure Evolution of Square-Shaped $\left(\mathrm{La}_{0.5} \mathrm{Ba}_{0.5}\right)\left(\mathrm{Mn}_{0.5} \mathrm{Fe}_{0.5}\right) \mathrm{O}_{3}$ Ceramic Nanoparticles. J. Ceram. Sci. Tech. 2015, 6, 221-230.

5. Nogués, J.; Sort, J.; Langlais, V.; Skumryev, V.; Surinach, S.; Munoz, J.S.; Baro, M.D. Exchange bias in nanostructures. Phys. Rep. 2005, 422, 65. [CrossRef]

6. Mahato, B.K.; Choudhury, S.; Mandal, R.; Barman, S.; Otani, Y.; Barman, A. Tunable configurational anisotropy in collective magnetization dynamics of $\mathrm{Ni}_{80} \mathrm{Fe}_{20}$ nanodot arrays with varying dot shapes. J. Appl. Phys. 2015, 117, 213909. [CrossRef]

7. Thevenard, L.; Zeng, H.T.; Petit, D.; Cowburn, R.P. Macrospin limit and configurational anisotropy in nanoscale permalloy triangles. J. Magn. Magn. Mater. 2010, 322, 2152-2156. [CrossRef]

8. Cowburn, R.P.; Adeyeye, A.O.; Welland, M.E. Configurational anisotropy in nanomagnets. Phys. Rev. Lett. 1998, 81, 5414. [CrossRef]

9. Wachowiak, A.; Wiebe, J.; Bode, M.; Pietzsch, O.; Morgenstern, M.; Wiesendanger, R. Direct Observation of Internal Spin Structure of Magnetic Vortex Cores. Science 2002, 298, 577-580. [CrossRef] [PubMed]

10. Guslienko, K.Y.; Aranda, G.R.; Gonzalez, J.M. Topological gauge field in nanomagnets: Spin-wave excitations over a slowly moving magnetization background. Phys. Rev. B 2010, 81, 014414. [CrossRef]

11. Zhang, W.; Haas, S. Phase diagram of magnetization reversal processes in nanorings. Phys. Rev. B 2010, 81, 064433. [CrossRef]

12. Zhu, F.Q.; Fan, D.L.; Zhu, X.C.; Zhu, J.G.; Cammarata, R.C.; Chien, C.L. Ultrahigh-Density Arrays of Ferromagnetic Nanorings on Macroscopic Areas. Adv. Mater. 2004, 16, 2155. [CrossRef]

13. Mejia-López, J.; Altbir, D.; Romero, A.H.; Batlle, X.; Roshchin, I.V.; Li, C.-P.; Schuller, I.K. Vortex state and effect of anisotropy in sub-100-nm magnetic nanodots. J. Appl. Phys. 2006, 100, 104319. [CrossRef]

14. Ehrmann, A.; Blachowicz, T. Influence of shape and dimension on magnetic anisotropies and magnetization reversal of Py, Fe, and Co nano-objects with four-fold symmetry. AIP Adv. 2015, 5, 097109. [CrossRef]

15. Subramani, A.; Geerpuram, D.; Domanowski, A.; Baskaran, V.; Metlushko, V. Vortex state in magnetic rings. Phys. C 2004, 404, 241. [CrossRef]

16. Wang, J.; Adeyeye, A.O.; Singh, N. Magnetostatic interactions in mesoscopic $\mathrm{Ni}_{80} \mathrm{Fe}_{20}$ ring arrays. Appl. Phys. Lett. 2005, 87, 262508. [CrossRef]

17. Gao, X.S.; Adeyeye, A.O.; Goolaup, S.; Singh, N.; Jung, W.; Castano, F.J.; Ross, C.A. Inhomogeneities in spin states and magnetization reversal of geometrically identical elongated Co rings. J. Appl. Phys. 2007, 101, 09F505. [CrossRef]

18. Vavassori, P.; Grimsditch, M.; Novosad, V.; Metlushko, V.; Ilic, B. Metastable states during magnetization reversal in square permalloy rings. Phys. Rev. B 2003, 67, 134429. [CrossRef]

19. Vavassori, P.; Bovolenta, R.; Metlushko, V.; Ilic, B. Vortex rotation control in Permalloy disks with small circular voids. J. Appl. Phys. 2006, 99, 053902. [CrossRef]

20. Leong, T.G.; Zarafshar, A.M.; Gracias, D.H. Three-dimensional fabrication at small size scales. Small 2010, 6, 792. [CrossRef]

21. Amaladass, E.; Ludescher, B.; Schütz, G.; Tyliszczak, T.; Lee, M.-S.; Eimüller, T. Nanospheres generate out-of-plane magnetization. J. Appl. Phys. 2010, 107, 053911. [CrossRef] 
22. Blachowicz, T.; Ehrmann, A.; Steblinski, P.; Pawela, L. Magnetization reversal in magnetic half-balls influenced by shape perturbations. J. Appl. Phys. 2010, 108, 123906. [CrossRef]

23. Ehrmann, A.; Blachowicz, T. Vortex and double-vortex nucleation during magnetization reversal in Fe nanodots of different dimensions. J. Magn. Magn. Mater. 2019, 475, 727-733. [CrossRef]

24. Lyberatos, A.; Komineas, S.; Papanicolaou, N. Precessing vortices and antivortices in ferromagnetic elements. J. Appl. Phys. 2011, 109, 023911. [CrossRef]

25. Ehrmann, A.; Blachowicz, T. Systematic study of magnetization reversal in square Fe nanodots of varying dimensions in different orientations. Hyperfine Interact. 2018, 239, 48. [CrossRef]

26. Mohler, G.; Harter, A.W. Micromagnetic investigation of resonance frequencies in ferromagnetic particles. J. Appl. Phys. 2005, 97, 10E313. [CrossRef]

27. Kanso, H.; Pate, R.; Baltz, V.; Ledue, D. Influence of finite-size and edge effects on the exchange-bias properties of ferromagnetic/antiferromagnetic nanodots: Granular Monte Carlo investigation. Phys. Rev. B 2019, 99, 054410. [CrossRef]

28. Depondt, P.; Levy, J.-C.S.; Mamica, S. Vortex polarization dynamics in a square magnetic nanodot. J. Phys. Cond. Matter 2013, 25, 466001. [CrossRef]

29. Ivanov, Y.P.; Nefedev, K.V.; Iljin, A.I.; Pustovalov, E.V.; Chebotkevich, L.A. Magnetization reversal of nanodots with different magnetic anisotropy and magnetostatic energy. J. Phys. Conf. Ser. 2011, 266, 012117. [CrossRef]

30. Song, S.Y.; Zhang, G.F.; Song, W.B.; Guo, G.H. Dynamical reversal of rectangular nanodot studied by micromagnetics. Act. Phys. Sin. 2009, 58, 5757-5762.

31. Tillmanns, A.; Blachowicz, T.; Fraune, M.; Güntherodt, G.; Schuller, I.K. Anomalous magnetization reveral mechanism in unbiased $\mathrm{Fe} / \mathrm{FeF}_{2}$ investigated by means of the magneto-optic Kerr effect. J. Magn. Magn. Mater. 2009, 321, 2932-2935. [CrossRef]

32. Nasirpouri, F.; Engbarth, M.A.; Bending, S.J.; Peter, L.M.; Knittel, A.; Fangohr, H.; Milosevic, M.V. Three-dimensional ferromagnetic architectures with multiple metastable states. Appl. Phys. Lett. 2011, 98, 222506. [CrossRef]

33. Mulkers, J.; Milosevic, M.V.; van Waeyenberge, B. Cycloidal versus skyrmionic states in mesoscopic chiral magnets. Phys. Rev. B 2016, 93, 214405. [CrossRef]

34. Mulkers, J.; van Waeyenberge, B.; Milosevic, M.V. Effects of spatially engineered Dzyaloshinskii-Moriya interaction in ferromagnetic films. Phys. Rev. B 2017, 95, 144401. [CrossRef]

35. Mulkers, J.; van Waeyenberge, B.; Milosevic, M.V. Tunable Snell's law for spin waves in heterochiral magnetic films. Phys. Rev. B 2018, 97, 104422. [CrossRef]

36. Menezes, R.M.; Mulkers, J.; de Souza Silva, C.C.; Milosevic, M.V. Deflection of ferromagnetic and antiferromagnetic skymions at heterochiral interfaces. Phys. Rev. B 2019, 99, 104409. [CrossRef]

37. Wang, S.Y.; Zhan, Q.W. Modified bow-tie antenna with strong broadband field enhancement for RF photonic applications. Proc. SPIE 2013, 8806, 88061V.

38. Ehrmann, A.; Blachowicz, T. Influence of the distance between nanoparticles in clusters on the magnetization reversal process. J. Nanomater. 2017, 2017, 5046076. [CrossRef]

39. Ehrmann, A.; Blachowicz, T. Interaction between magnetic nanoparticles in clusters. AIMS Mater. Sci. 2017, 4, 383-390. [CrossRef]

40. Donahue, M.J.; Porter, D.G. OOMMF User's Guide, Version 1.0; Interagency Report NISTIR 6376; National Institute of Standards and Technology: Gaithersburg, MD, USA, 1999.

41. Gilbert, T.L. A phenomenological theory of damping in ferromagnetic materials. IEEE Trans. Magn. 2004, 40, 3443. [CrossRef]

42. Leliart, J.; Dvornik, M.; Mulkers, J.; de Clercq, J.; Milosevic, M.V.; van Waeyenberge, B. Fast micromagnetic simulations on GPU—Recent advances made with mumax ${ }^{3}$. J. Phys. D Appl. Phys. 2018, 51, 123002. [CrossRef]

43. Oezelt, H.; Kirk, E.; Wohlhüter, P.; Müller, E.; Heyerman, L.J.; Kowacs, A.; Schrefl, T. Vortex motion in amorphous ferrimagnetic thin film elements. AIP Adv. 2017, 7, 056001. [CrossRef]

(C) 2020 by the authors. Licensee MDPI, Basel, Switzerland. This article is an open access article distributed under the terms and conditions of the Creative Commons Attribution (CC BY) license (http://creativecommons.org/licenses/by/4.0/). 